\title{
Modeling of relative intensity noise and terminal electrical noise of semiconductor lasers using artificial neural network
}

\author{
A. $\operatorname{Rezaei}^{1} \cdot$ L. Noori ${ }^{1}$
}

Received: 8 April 2015/Accepted: 31 May 2016/Published online: 8 June 2016

(C) The Author(s) 2016. This article is published with open access at Springerlink.com

\begin{abstract}
In this paper, artificial neural network (ANN) is used to predict the source laser's relative intensity noise (RIN) and the terminal electrical noise (TEN) of semiconductor lasers. For this purpose, the multi-layer perceptron (MLP) neural network trained with the back propagation algorithm is used. To develop this model, the normalized bias current and frequency are selected as the input parameters and the RIN and TEN of semiconductor lasers are selected as the output parameters. The obtained results show that the proposed ANN model is in a good agreement with the numerical method, and a small error between the predicted values and the numerical solution is obtained. Therefore, the proposed ANN model is a useful, reliable, fast and cheap tool to predict the RIN and TEN of semiconductor lasers.
\end{abstract}

Keywords Artificial neural network · Multi-layer perceptron $\cdot$ Relative intensity noise $\cdot$ Terminal electrical noise $\cdot$ Bias current

\section{Introduction}

The semiconductor lasers with the low relative intensity noise (RIN) and low terminal electrical noise (TEN) play an important role in the optical communication systems such as the subcarrier multiplexed (SCM) transmission systems, high-speed digital transmissions and the other optical microwave and millimeter-wave applications [1].

\section{Noori}

unrezaei@yahoo.com; Leila_noori62@yahoo.com

1 Electrical Engineering Department, Kermanshah University of Technology, Kermanshah, Iran
Based on the noise-equivalent model, two nonlinear equations for the RIN and TEN of semiconductor lasers have been obtained to present the effects of the normalized bias current and frequency [1]. To calculate the system noise, one method is the use of the equivalent circuit model of the noise. In addition, the rate equations can be used to calculate the laser noise, including a noise term named Langevin noise [2-6]. In [2] the noise equivalent circuit of the laser diode has been obtained as a function of the bias current and frequency. In [7], the intensity noise in semiconductor lasers has been analyzed using a systematic technique, where the Langevin noise sources have been obtained and the laser rate equations have been derived. To predict the performances of an optical fiber link, the simulation results of this optical fiber link have been presented using ADS simulator in [8]. The obtained results in [8] show that the effect of the laser diode relative intensity noise is great. In [9] using the rate equations, a semiclassical noise model for the RIN has been obtained and its properties have been analyzed by adding individual noise sources contributing to the RIN. To predict the behavior of the TEN and RIN, two nonlinear equations must be solved [1]. Also, for all normalized bias currents between a certain frequency range, the behavior of the RIN and TEN has not been achieved. In this paper, the behavior of the RIN and TEN are modeled and predicted using artificial neural network (ANN) as a function of the normalized bias current and frequency. Because of the ability of ANNs in extracting the information from the nonlinear and noisy data, an ANN model is applied to predict the RIN and TEN of semiconductor lasers. This method has many advantages such as reliability, accuracy, time saving and high efficiency. The numerical method presented in [1] is used to provide the training and testing samples for the proposed 
ANN model, then, the behavior of the RIN and TEN are analyzed using the trained ANN model in a wide range of the normalized bias current and frequency. It will be shown that the results of the proposed ANN model are very close to the numerical method.

\section{Artificial neural network modeling}

The noise-equivalent circuit model for the semiconductor lasers can be obtained from the rate equation. Using the noise-equivalent circuit model, the TEN and RIN can be given by [1]:

$\mathrm{TEN}=\frac{\left\langle i_{n 1}^{2}\right\rangle\left[R_{S}^{2}+\left(\omega L_{S}\right)^{2}\right]+\left\langle i_{n 2}^{2}\right\rangle R_{S}^{2}+2\left\langle i_{n 1} i_{n 2}^{*}\right\rangle R_{S}^{2}}{\left(L_{S} C_{t} q G_{0}\right)^{2} D D^{*}}$

$$
\mathrm{RIN}=\frac{\left\langle i_{n 1}^{2}\right\rangle+\left\langle i_{n 2}^{2}\right\rangle R_{S}^{2}\left[1 / R_{d}^{2}+\left(\omega C_{t}\right)^{2}\right]-2\left\langle i_{n 1} i_{n 2}^{*}\right\rangle R_{S} / R_{j}}{\left(L_{S} C_{t} q G_{0}\right)^{2} D D^{*}}
$$

where, $R_{S}, R_{d}, L_{S}$, and $C_{t}$ are the resistances, inductance, and capacitance of the linear noiseless laser network, respectively. Also, $i_{n 1}$ and $i_{n 2}$ are the noise current sources in the input and output ports of the laser diode noise equivalent circuit model, respectively and:

$D=\left[\frac{1}{L_{S} C_{t}}\left(1+\frac{R_{S}}{R_{j}}\right)-\omega^{2}\right]+j \omega\left(\frac{R_{S}}{L_{S}}+\frac{1}{R_{J} C_{t}}\right)$

$G_{0}=1.2 \times 10^{-12}\left(N_{o}-N_{\text {om }}\right) ;$ and $N_{\text {om }}=1.45 \times 10^{24}$

Based on Eqs. (1.a) and (1.b), two nonlinear equations for the TEN and RIN are obtained. For the different frequencies $\omega$, the numerical data of the TEN and RIN can be achieved as the functions of the normalized bias current [1]. These data describe the TEN and RIN for only limited values of the normalized bias current.

To predict the behavior of the TEN and RIN, an ANN model can be used. ANN is a good way to handle the problems of modeling, prediction, and control. ANN has been used in different applications such as engineering, medicine, and finance. The fundamental processing element of ANN is the neuron. Multi-layer perceptron (MLP) is the most widely used ANN. MLP network has one input layer, one or more hidden layers and one output layer with at least one neuron in each layer. In this Paper, MLP network is used to model and predict the TEN and RIN. The proposed MLP model is shown in Fig. 1, where the inputs are the normalized bias current and frequency and the outputs are the RIN and TEN given in Eqs. (1.a) and (1.b).

The output from $i$ th neuron of the hidden layer is given by [10]: $\beta_{i}=f\left(\sum_{k=1}^{2}\left(x_{k} W_{k i}\right)+b_{i}\right) i=1,2, \ldots, 5$

where, $x$ is the input, $b$ is the bias term, $W$ is the weighting factor and $f$ for variable $\mathrm{u}$ is defined as follows:

$f(u)=\operatorname{Tansig}(u)=\frac{2}{1+e^{-2 u}}-1$

The output of the $j$ th neuron in the output layer is given by:

$y_{j}=\sum_{k=1}^{5}\left(\beta_{k} W_{k j}\right)+b_{j} \quad j=1,2$

The data set required for the training and testing of the proposed ANN model is obtained from Ref. [1]. To develop the model, total data are divided into two

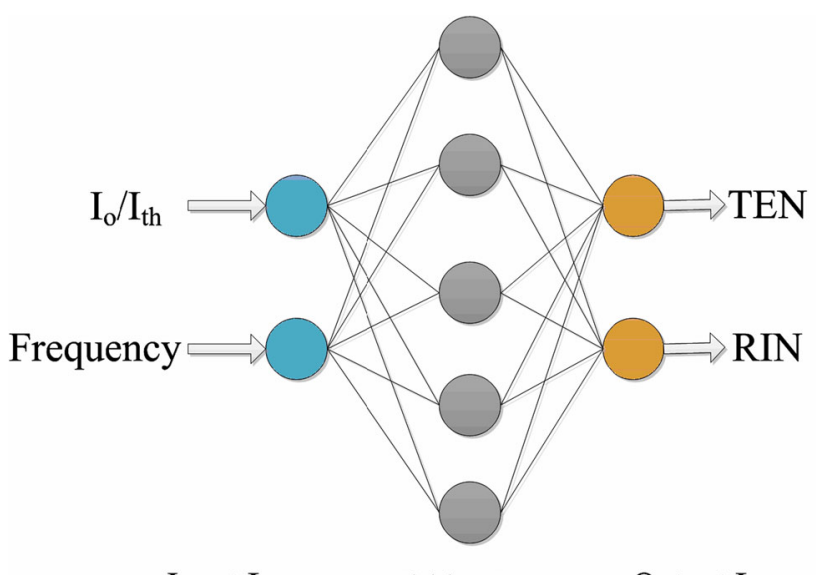

Input Layer Hidden Layer Output Layer

Fig. 1 The proposed ANN model

Table 1 Characteristics of the proposed ANN model

\begin{tabular}{ll}
\hline Neural network & MLP \\
\hline Number of hidden layer & 1 \\
Number of neurons in the input layer & 2 \\
Number of neurons in the hidden layer & 5 \\
Number of neurons in the output layer & 2 \\
Learning rate & 0.5 \\
Number of epochs & 100 \\
Adaption learning function & Trainlm \\
Activation function & Tansig \\
\hline
\end{tabular}

Table 2 The obtained MRE \% for the proposed ANN model

\begin{tabular}{lll}
\hline MRE \% & Train & Test \\
\hline TEN & 0.86 & 1.62 \\
RIN & 0.61 & 0.71 \\
\hline
\end{tabular}


Table 3 Comparison between the numerical and predicted (ANN) results for the testing data

\begin{tabular}{|c|c|c|c|c|c|}
\hline \multirow[t]{2}{*}{$I_{0} / I_{\mathrm{th}}$} & \multirow[t]{2}{*}{$F(\mathrm{GHz})$} & \multicolumn{2}{|l|}{ Numerical } & \multicolumn{2}{|l|}{ ANN } \\
\hline & & TEN (dB) & RIN (dB) & TEN (dB) & $\mathrm{RIN}(\mathrm{dB})$ \\
\hline 1 & 0.1 & -119 & -178 & -114.87 & -177.55 \\
\hline 1 & 0.602 & -105 & -178 & -108.16 & -179.36 \\
\hline 1 & 0.14 & -116 & -178 & -114.18 & -177.63 \\
\hline 1 & 0.396 & -108 & -178 & -110.38 & -178.38 \\
\hline 1 & 0.798 & -104 & -180 & -106.91 & -180.81 \\
\hline 1 & 1.99 & -111 & -194 & -109.72 & -191.44 \\
\hline 1 & 3.02 & -116 & -201 & -115.46 & -201.85 \\
\hline 1 & 4.44 & -119 & -208 & -118.71 & -208.4 \\
\hline 1 & 6.97 & -123 & -216 & -124.43 & -216.16 \\
\hline 1 & 9.56 & -126 & -222 & -127.39 & -219.87 \\
\hline 1.5 & 0.292 & -145 & -188 & -145.8 & -187.35 \\
\hline 1.5 & 1 & -133 & -187 & -132.68 & -184.44 \\
\hline 1.5 & 1.48 & -128 & -185 & -126.31 & -183.14 \\
\hline 1.5 & 2.67 & -103 & -166 & -105.23 & -168.25 \\
\hline 1.5 & 3.02 & -114 & -176 & -111.65 & -175.72 \\
\hline 1.5 & 6.97 & -133 & -203 & -128.58 & -201.81 \\
\hline 2 & 0.204 & -156 & -191 & -156.03 & -192.93 \\
\hline 2 & 0.602 & -148 & -191 & -148.59 & -191.29 \\
\hline 2 & 1.48 & -139 & -190 & -137.11 & -188.76 \\
\hline 2 & 1.99 & -135 & -189 & -132.69 & -187.8 \\
\hline 2 & 3.02 & -125 & -183 & -126.65 & -186.21 \\
\hline 2 & 4.44 & -122 & -182 & -118.44 & -178.35 \\
\hline
\end{tabular}

categories. One set for training (about $75 \%$ ) and the rest for testing (about $25 \%$ ). Table 1 shows the characteristics of the proposed ANN model.

\section{Results and discussions}

The training and testing results of the proposed ANN model in comparison with the numerical results [1] are shown in Tables 2, 3 and Fig. 2. Figures 3 and 4 compare

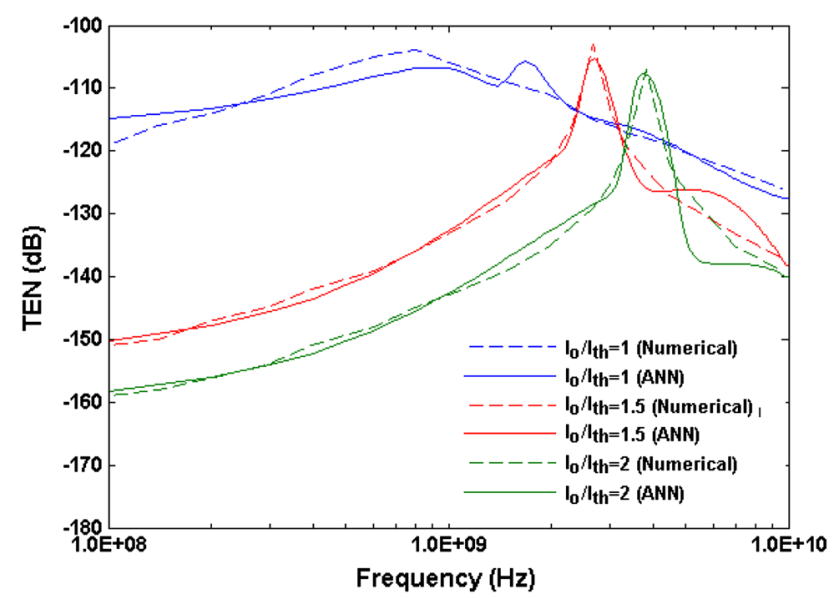

Fig. 3 Comparison between the numerical [1] and predicted results using the proposed ANN model for the TEN
Fig. 2 Comparison between the proposed ANN model and numerical results for the training and testing data
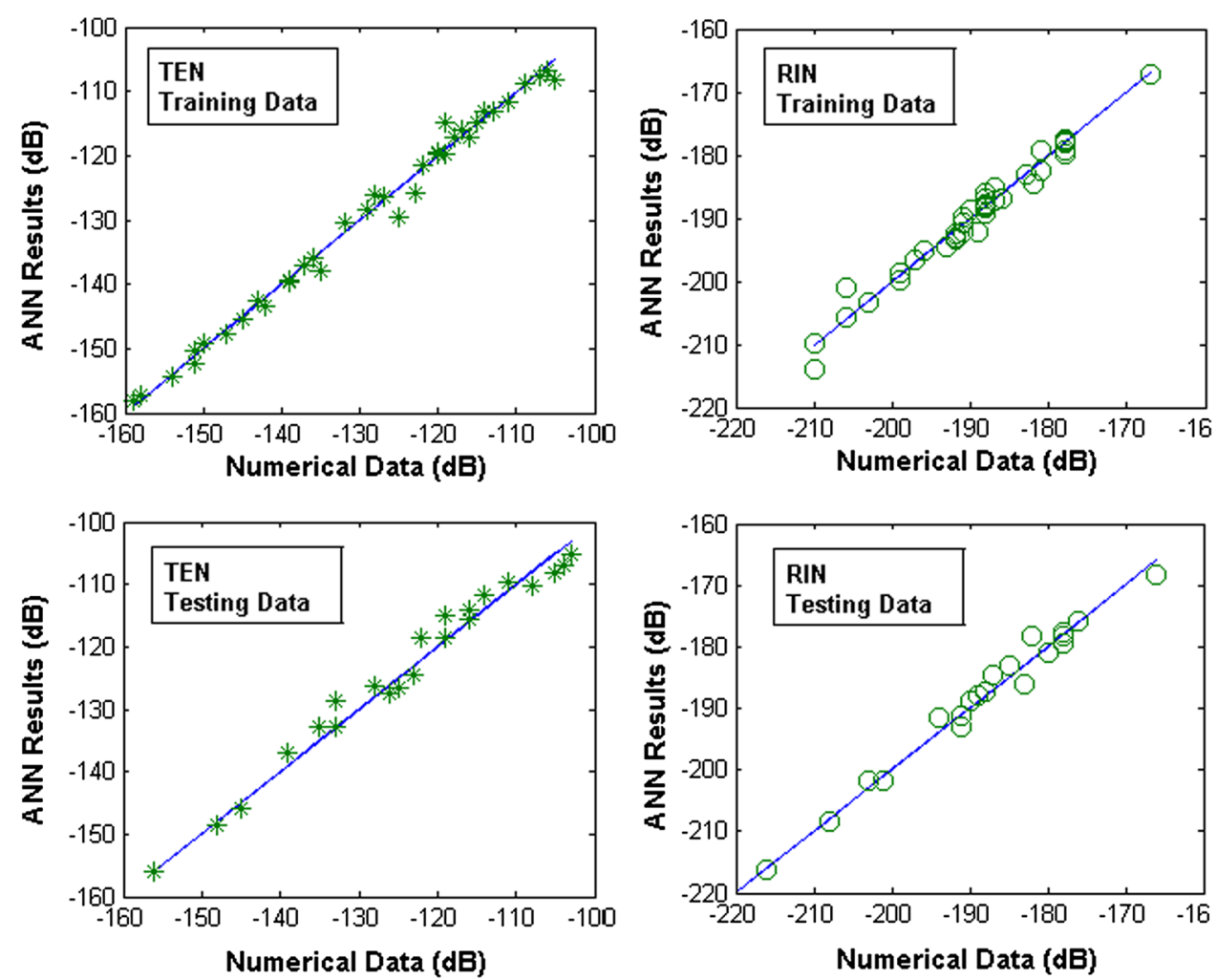


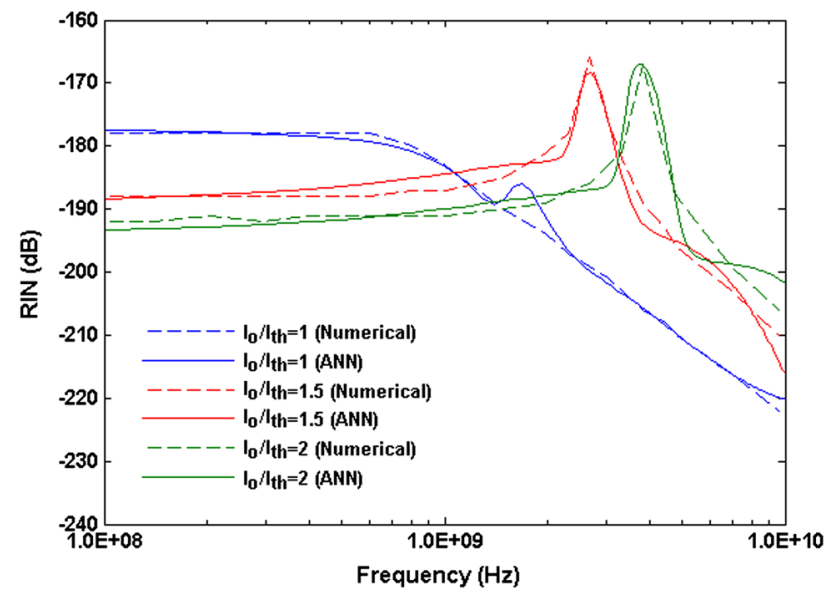

Fig. 4 Comparison between the numerical [1] and predicted results using the proposed ANN model for the RIN

the obtained results of the proposed ANN model and the numerical solution [1].

In Table 2, the mean relative error percentage (MRE \%) is calculated by:

$M R E \%=100 \times \frac{1}{N} \sum_{i=1}^{N}\left|\frac{X_{i}(\operatorname{Exp})-X_{i}(\operatorname{Pr} e d)}{X_{i}(\operatorname{Exp})}\right|$ where, $\mathrm{N}$ is the number of data and ' $X(\mathrm{Num})$ ' and ' $X($ Pred $)$ ' stand for the numerical values obtained in [1], and the predicted (ANN) values, respectively. Figures 5 and 6 show the TEN and RIN values at the different frequencies for all values of the $I_{\mathrm{o}} / I_{\mathrm{th}}$. These curves are obtained using the proposed ANN model. Finally, the proposed ANN model can present a mathematical relationship for the TEN and RIN to directly solve their equations as shown in Table 4.

From these results, it is clear that there is a good agreement between the numerical and predicted values for the output parameters. Therefore, the proposed ANN model can be used as an accurate model to predict the behavior of the RIN and TEN.

\section{Conclusion}

In this paper, the behavior of the relative intensity noise and terminal electrical noise as a function of the normalized bias current and frequency is modeled and predicted using artificial neural network. The proposed ANN model is in a good agreement with the numerical data with a minimum error. Also, the proposed ANN model presented a mathematical relationship for the TEN and RIN to
Fig. 5 The obtained results using the proposed ANN model for the TEN

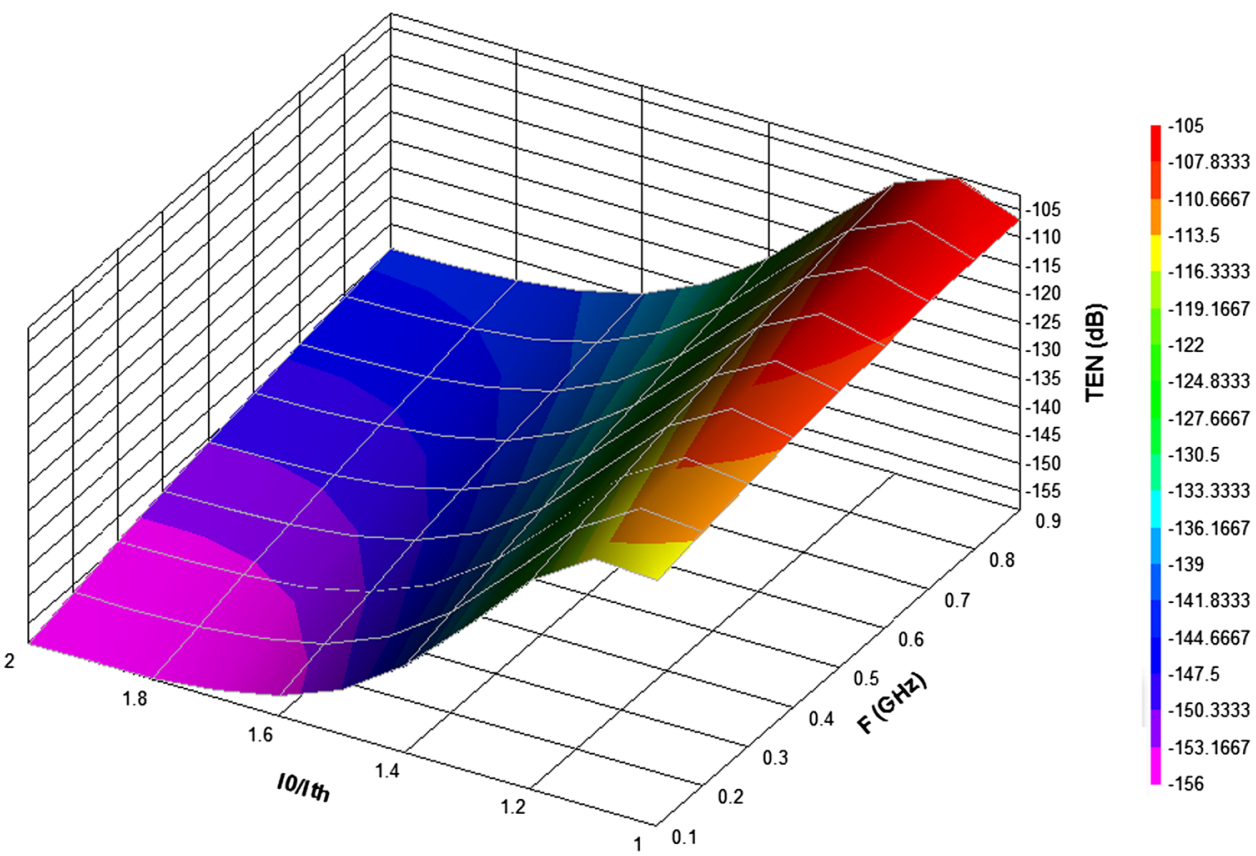


Fig. 6 The obtained results using the proposed ANN model for the RIN

Table 4 The obtained equations for the TEN and RIN using the proposed ANN model

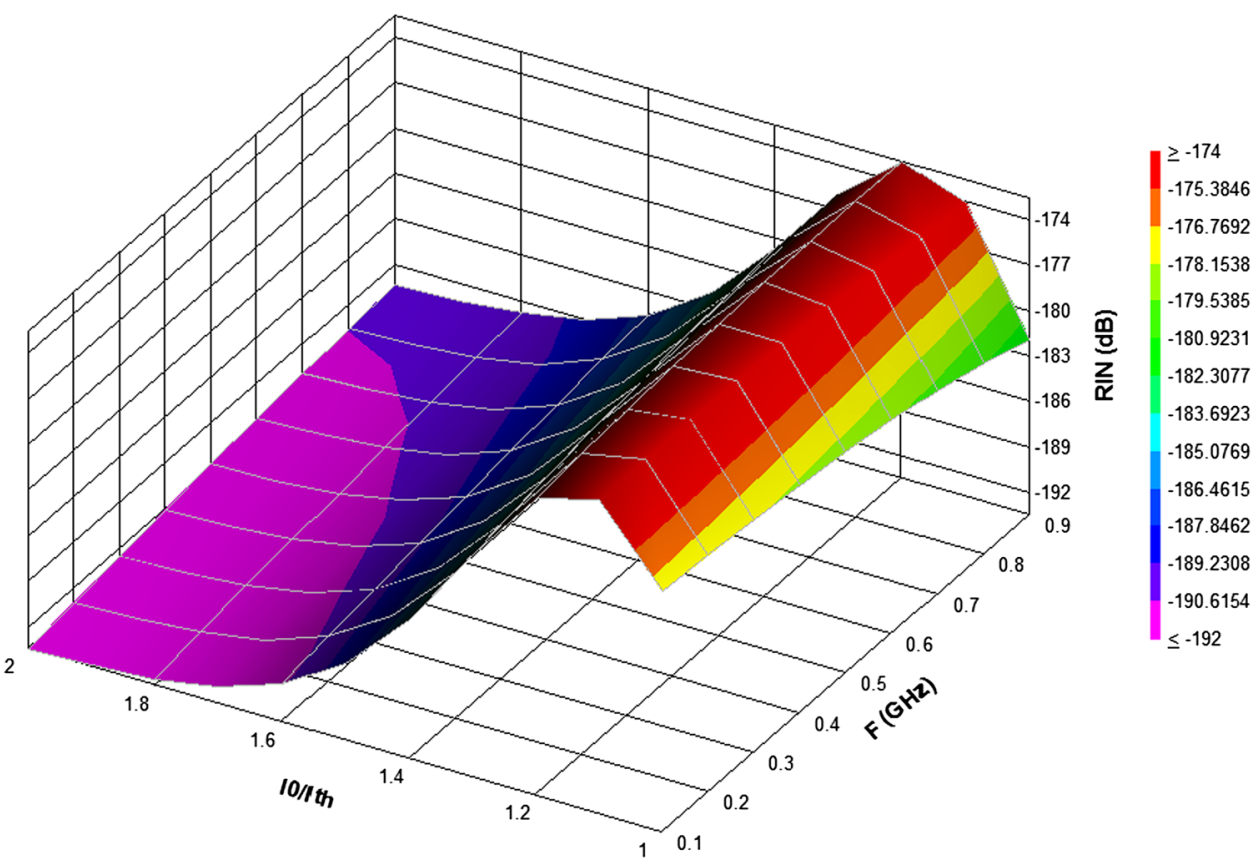

\begin{tabular}{|c|c|c|}
\hline $\mathrm{W} 13=-9.96$ & $\mathrm{~W} 27=0.128$ & $\mathrm{~W} 68=-1.74$ \\
\hline $\mathrm{W} 14=5.74$ & $\mathrm{~B} 3=1.51$ & $\mathrm{~W} 78=1.79$ \\
\hline $\mathrm{W} 15=-0.060$ & $\mathrm{~B} 4=-2.69$ & $\mathrm{~W} 39=0.104$ \\
\hline $\mathrm{W} 16=-3.04$ & $\mathrm{~B} 5=-1.41$ & $\mathrm{~W} 49=0.167$ \\
\hline $\mathrm{W} 17=-3.16$ & $\mathrm{~B} 6=3.190$ & $\mathrm{~W} 59=-0.999$ \\
\hline $\mathrm{W} 23=5.47$ & $\mathrm{~B} 7=3.57$ & $\mathrm{~W} 69=-1.40$ \\
\hline $\mathrm{W} 24=-1.93$ & $\mathrm{~W} 38=0.094$ & $\mathrm{~W} 79=1.28$ \\
\hline $\mathrm{W} 25=-0.30$ & $\mathrm{~W} 48=0.175$ & $\mathrm{~B} 8=-5.76$ \\
\hline $\mathrm{W} 26=0.152$ & $\mathrm{~W} 58=-4.51$ & $\mathrm{~B} 9=-3.03$ \\
\hline \multicolumn{3}{|c|}{$\mathrm{Y} 1=\operatorname{Tansig}(\mathbf{I O I T H} * \mathrm{~W} 13+\mathbf{F R E Q} * \mathrm{~W} 23+\mathrm{B} 3)$} \\
\hline \multicolumn{3}{|c|}{ Y2 $=$ Tansig $(\mathbf{I O I T H} * \mathrm{~W} 14+$ FREQ $*$ W24 + B4) } \\
\hline \multicolumn{3}{|c|}{ Y3 $=$ Tansig $(\mathbf{I O I T H} * \mathrm{~W} 15+$ FREQ $*$ W25 + B5) } \\
\hline \multicolumn{3}{|c|}{ Y4 = Tansig $(\mathbf{I O I T H} * \mathrm{~W} 16+$ FREQ $*$ W26 + B6) } \\
\hline \multicolumn{3}{|c|}{ Y5 $=$ Tansig $(\mathbf{I O I T H} * \mathrm{~W} 17+$ FREQ $*$ W27 + B7) } \\
\hline \multicolumn{3}{|c|}{ TEN $=100 *(\mathrm{Y} 1 * \mathrm{~W} 38+\mathrm{Y} 2 * \mathrm{~W} 48+\mathrm{Y} 3 * \mathrm{~W} 58+\mathrm{Y} 4 * \mathrm{~W} 68+\mathrm{Y} 5 * \mathrm{~W} 78+\mathrm{B} 8)$} \\
\hline \multicolumn{3}{|c|}{$\mathbf{R I N}=100 *(\mathrm{Y} 1 * \mathrm{~W} 39+\mathrm{Y} 2 * \mathrm{~W} 49+\mathrm{Y} 3 * \mathrm{~W} 59+\mathrm{Y} 4 * \mathrm{~W} 69+\mathrm{Y} 5 * \mathrm{~W} 79+\mathrm{B} 9)$} \\
\hline
\end{tabular}

directly solve their equations. According to the results obtained from the proposed model, it can be seen that the ANN model can be used to model and predict the output with a high accuracy and saving time and money.

Open Access This article is distributed under the terms of the Creative Commons Attribution 4.0 International License (http://crea tivecommons.org/licenses/by/4.0/), which permits unrestricted use, distribution, and reproduction in any medium, provided you give appropriate credit to the original author(s) and the source, provide a link to the Creative Commons license, and indicate if changes were made.

\section{References}

1. Gao, J.: Optoelectronic Integrated Circuit Design and Device Modeling. East China Normal University, Shanghai (2011)

2. Harder, C., Katz, J., Margalit, S.: Noise equivalent circuit of a semiconductor laser diode. IEEE J. Quantum Electron. 18(3), 333-337 (1982)

3. Andrekson, P.A.: Abdersson, P, Alping, A: In situ characterization of laser diodes from wide band electrical noise measurements. J. Lightwave Technol. 4(7), 804-811 (1986)

4. Orsal, B., Signoret, P., Peransin, J.M.: Correlation between electrical and optical photocurrent noises in semiconductor laser diode. IEEE Trans. Electron. Dev. 4(11), 2151-2160 (1994) 
5. Bich-Ha, T.T., Mollier, J.: Noise equivalent circuit of a two mode semiconductor laser with the contribution of both the linear and the nonlinear gain. IEEE J. Sel. Top. Quantum Electron. 3(4), 304-308 (1997)

6. Mortazy, E., Ahmadi, V., MK, Moravvej Farshi: An integrated equivalent circuit model for relative intensity noise and frequency noise spectrum of a multimode semiconductor laser. IEEE J. Quantum Electron. 38(10), 1366-1371 (2002)

7. Ahmed, M., Yamada, M., Saito, M.: Numerical modeling of intensity and phase noise in semiconductor lasers. IEEE J. Quantum Electron. 37(12), 1600-1610 (2001)
8. Algani. C, Billabert. A, Deshours. F, Chettat. H, Rumelhard. C, Blache.F, Alquie. G.: Main noise influence of the RIN laser diode of an EML transmitter used in an UWB RoF link. Microwave Conference (EuMC), 117-120, (2010)

9. Gensty T., Elsaber W.: Semiclassical model for the relative intensity noise of intersub band quantum cascade lasers. Elsevier, Optics Communications. 256, 171-183, (2005)

10. Taylor, J.G.: Neural networks and their applications. Wiley, West sussex (1996) 